\title{
Longitudinal emittance blowup in fixed field alternating gradient muon accelerators
}

\author{
Shinji Machida* \\ ASTeC, CCLRC Rutherford Appleton Laboratory, Chilton, Didcot, Oxfordshire, OX11 OQX, United Kingdom
}

(Received 1 October 2006; published 27 October 2006)

\begin{abstract}
The fixed field alternating gradient (FFAG) accelerator is proposed as a muon accelerator because of its large aperture and no need of magnet ramping. In particular, the nonscaling type of FFAG has been studied because of its simple magnets and its unique acceleration method using the path out of the rf bucket. A recent $6 \mathrm{D}$ tracking study reveals, however, that the time of flight difference due to transverse amplitude causes the longitudinal emittance blowup which limits the transverse acceptance. This is a serious problem for an accelerator that is expected to accelerate a muon beam with huge transverse emittance. Two methods of curing the problem are examined by particle tracking. One is higher rf voltage and the other is higher harmonic rf in addition to the fundamental one. A $50 \%$ increase of rf voltage suppresses the emittance blowup. Second and third harmonic rf also improve the final momentum spread. Both methods work fine in a single FFAG system. However, a few percent of particle loss is inevitable when two FFAG are cascaded.
\end{abstract}

DOI: 10.1103/PhysRevSTAB.9.104002

PACS numbers: 29.20. $-\mathrm{c}, 41.75 .-\mathrm{i}$

\section{INTRODUCTION}

The fixed field alternating gradient (FFAG) has been considered as a muon accelerator for a neutrino factory. The FFAG comprises static magnets so that the acceleration can be very quick. Time required to complete an acceleration cycle is only limited by total rf voltage in a ring. Unlike the recirculating linear accelerator, a single FFAG arc accommodates a beam of a large momentum range from injection to extraction.

The idea of the FFAG as a muon accelerator was first proposed for use in a neutrino factory in Japan [1]. After the success of the proof of principle proton FFAG [2], they designed a four FFAG cascade to accelerate muons from 0.3 to $20 \mathrm{GeV}$. The type of FFAG in the design report is called a scaling FFAG in comparison with a newly proposed nonscaling FFAG [3]. Since the magnetic field is constant, the orbit moves as a beam is accelerated. However, each orbit corresponding to different momenta has a similar shape and the machine tune is kept constant in a whole cycle due to the cardinal or scaling condition [4]. In common accelerator terminology, chromaticity is zero in a whole cycle.

Although radial orbit excursion during acceleration is squeezed by the sharply rising magnetic field, magnets of scaling FFAG are still large mostly due to the scaling condition. The radial orbit excursion on the low momentum side particularly tends to be larger. This results in a costly design. Nonscaling FFAG can overcome those problems. For the application of FFAG to muon acceleration, a muon beam has to be accelerated very quickly against the muon lifetime. If the total turn number in a ring is the order of 10 , we probably do not have to worry about resonances and its crossing by a beam. Then, instead of satisfying the

*Electronic address: s.machida@rl.ac.uk scaling condition to make a tune constant, one can design optics such that the radial orbit excursion becomes minimum. It is also good to have only quadrupoles and dipoles so that a machine is, in principle, free from nonlinear resonances.

Muon acceleration by nonscaling FFAG has been adopted in a neutrino factory design [5] and many studies have been done [6]. One of the major issues is the $\mathrm{rf}$ acceleration scheme. Although a muon beam reaches almost the speed of light when it is injected to a FFAG accelerator at $5 \mathrm{GeV}$ or above, path length difference makes the revolution frequency change during acceleration. The rf accelerating frequency, on the other hand, cannot be synchronized because the change is so fast. A solution is to use a flow outside an rf bucket of a fixed frequency in the longitudinal phase space. If one designs a lattice such that the time of flight changes with parabolic shape and has the minimum in the energy between the low and high limits, one can keep a beam always around a crest of rf wave. That is often called "gutter acceleration" because the trajectory in the longitudinal phase space looks like flow in a gutter. Muon acceleration by FFAG becomes more plausible with those ingredients, namely, nonscaling FFAG optics and an out of bucket acceleration $[7,8]$.

The studies up to now look at beam dynamics in transverse and longitudinal directions separately and parameters are optimized independently. For acceleration of a muon beam, which has huge $100 \%$ normalized emittance such as $30 \pi \mathrm{mm}$ rad and large momentum spread of a few percent, coupling effects between transverse and longitudinal may not be negligible and that is the subject of this paper. One reason why such a study did not exist before is that there was not an adequate tracking code. Unlike a synchrotron, the central orbit of the FFAG moves as a beam is accelerated. A single truncated polynomial series, which most tracking codes for a synchrotron assume, does not 
suffice to represent optics because deviation from reference coordinates becomes large. For example, when the ratio of injection and extraction momenta is two, momentum swing is from $-33 \%$ to $+33 \%$. Conventional accelerator tracking codes fail to model FFAG [9]. The exception is PTC written by Forest [10]. Another difficulty in modeling FFAG with conventional accelerator codes is the mixture of magnet geometry and a beam orbit. In a nonscaling FFAG, for example, any orbit does not go through the center of quadrupole magnets. Positioning of lattice elements has to be totally separated from a beam orbit. This is not the case in PTC.

We have made a tracking code specifically for FFAG and studied $6 \mathrm{D}$ motion of a muon acceleration ring. It was found that the longitudinal phase space is distorted due to a finite transverse amplitude. Above a certain transverse amplitude, the particle cannot be accelerated. First, we show the tracking results of $6 \mathrm{D}$ motions. Second, we discuss possible cures. Third, we demonstrate the beam acceleration when two FFAG are cascaded. Discussions and a summary follow at the end.

\section{LONGITUDINAL EMITTANCE BLOW UP DUE TO TRANSVERSE AMPLITUDE}

Since a muon beam already reaches almost the speed of light when it is injected to the FFAG at $5 \mathrm{GeV}$ or above, the revolution frequency only depends on path length. In the fixed magnetic field in a FFAG, path length changes as a beam is accelerated. In addition, path length also depends on betatron amplitude. Suppose that there are two particles with zero and finite betatron amplitude. A zero amplitude particle goes straight in a ring, but a finite amplitude one takes a longer path to complete one revolution. In fact, Berg pointed out that the path length difference is proportional to chromaticity and betatron amplitude [11].

In nonscaling FFAG, first, the betatron amplitude is huge compared to that in an ordinary accelerator. For instance, the neutrino factory design report [5] states that $100 \%$ normalized muon emittance is $30 \pi \mathrm{mm} \mathrm{rad}$, whereas the typical value of proton emittance in a synchrotron is the order of $0.1 \pi \mathrm{mm}$ rad. Second, a nonscaling FFAG consists of only quadupoles and dipoles and, therefore, there is always finite chromaticity. These facts exaggerate the path length difference and therefore the time of flight to complete one revolution. Figure 1 shows the time of flight as a function of kinetic energy taking transverse amplitude as a parameter. Machine and beam parameters are taken from the 10 to $20 \mathrm{GeV}$ muon ring [12].

Another fact that makes things even worse is that there is no synchrotron oscillation in longitudinal phase space. With a scheme of acceleration out of bucket, there is no chance that a particle that lags behind recovers energy afterward. Thus, a large transverse amplitude particle will tend not to have adequate energy gain and some will not reach the final energy. Single-particle trajectories start-

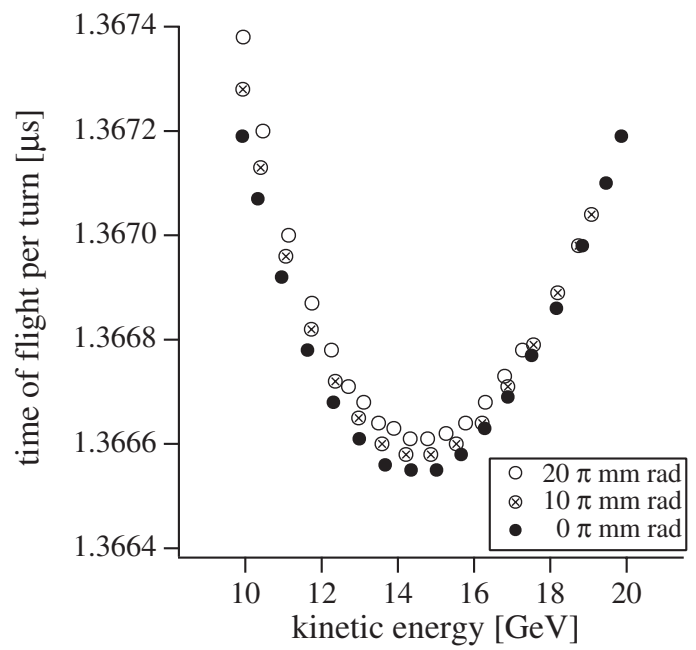

FIG. 1. Time of flight during acceleration for different transverse amplitude.

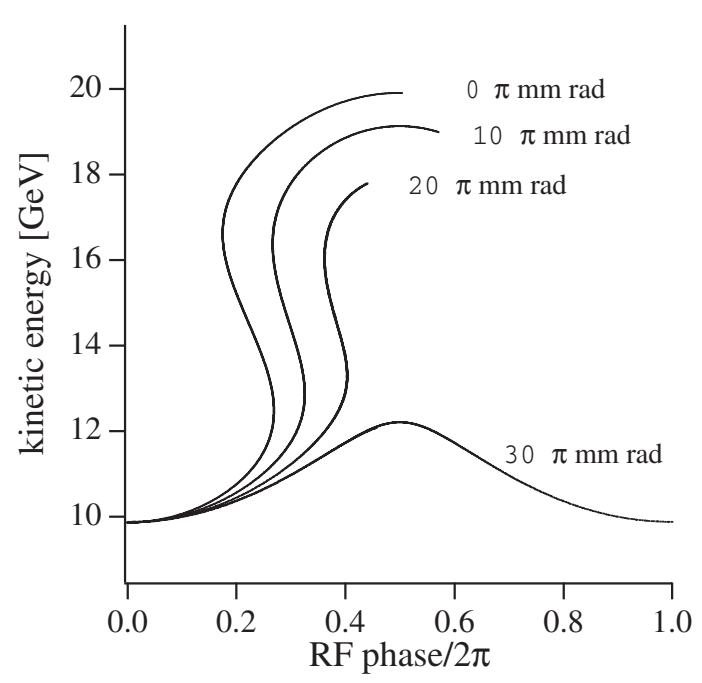

FIG. 2. Single-particle trajectory in longitudinal phase space. All particles start from the same point but with different transverse amplitude.

ing from the same place in longitudinal phase space but with different transverse amplitudes are shown in Fig. 2. A particle with single-particle emittance of $20 \pi \mathrm{mm} \mathrm{rad}$ in both horizontal and vertical planes is barely accelerated to the final energy in that example. Figures 3(a) and 3(b) show the evolution of a bunch without and with transverse distribution, respectively. It is manifest that finite transverse emittance causes longitudinal emittance blowup.

\section{INITIAL PARTICLE DISTRIBUTION}

The longitudinal emittance blowup also depends on how the initial particle distribution is made. Figure 3(b) assumes that there is no correlation between longitudinal and transverse phase space at the beginning. Longitu- 


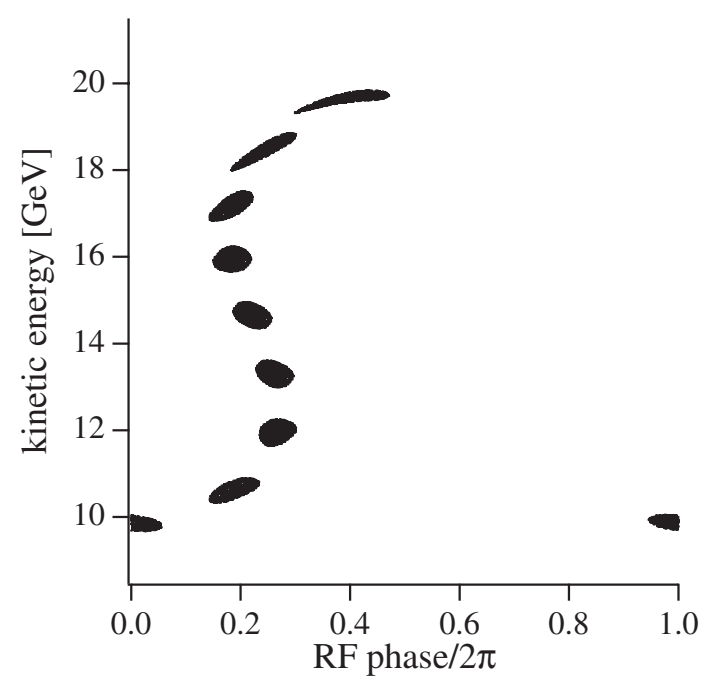

(a)

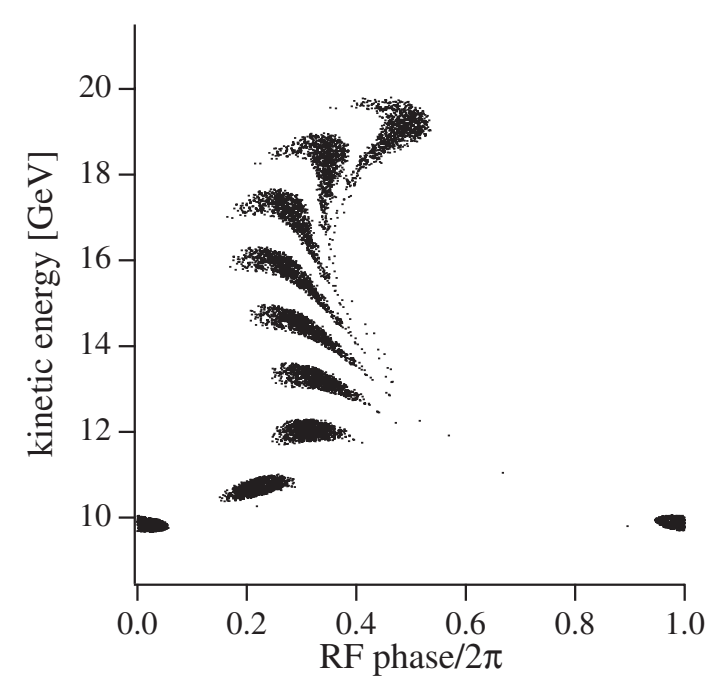

(b)

FIG. 3. Longitudinal emittance evolution (a) without and (b) with $100 \%$ transverse emittance of $30 \pi \mathrm{mm} \mathrm{rad}$.

dinally, a beam with emittance of $0.5 \mathrm{eV} s$ is created and the particle distribution inside is uniform. Its shape is matched according to Berg's criterion [13]. Independently, initial particles are prepared in the transverse 4D ellipsoidal phase space with Waterbag distribution.

The blowup is somewhat suppressed if there is a correlation between longitudinal and transverse distribution. In Fig. 4(a), longitudinal and transverse emittance are the same as before but all the initial particles are within 6D ellipsoidal phase space. Particles with large amplitude in both longitudinal and transverse phase space are elimi- nated. On the other hand, the blowup is more pronounced when there is no correlation between horizontal and vertical phase space as shown in Fig. 4(b). When initial particles are generated in longitudinal, horizontal, and vertical phase space independently, the blowup becomes largest.

A question is which initial distribution is most realistic. Emittance right after a pion production target is expected to be huge and no correlation exists. However, not all the particles are transported to the entrance of a FFAG. Transverse emittance is likely to be collimated by the aperture of upstream machines. With a circular shape of

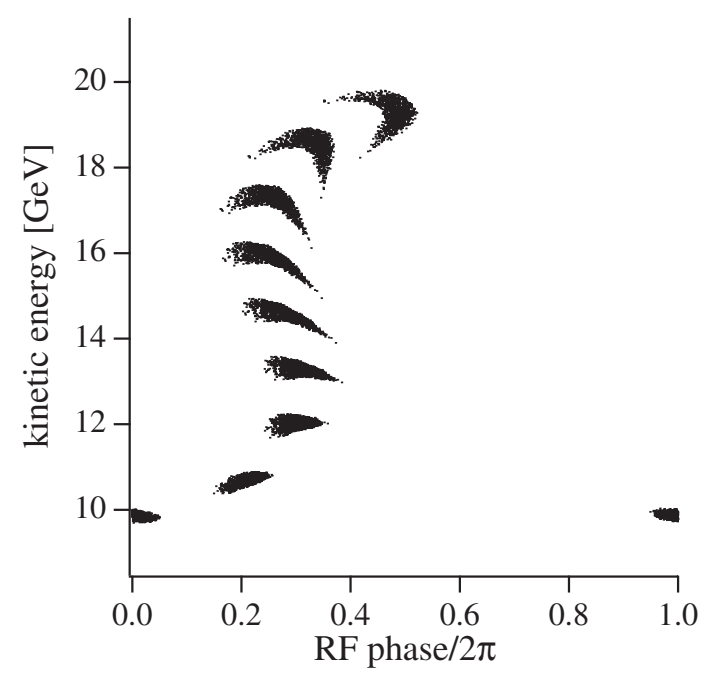

(a)

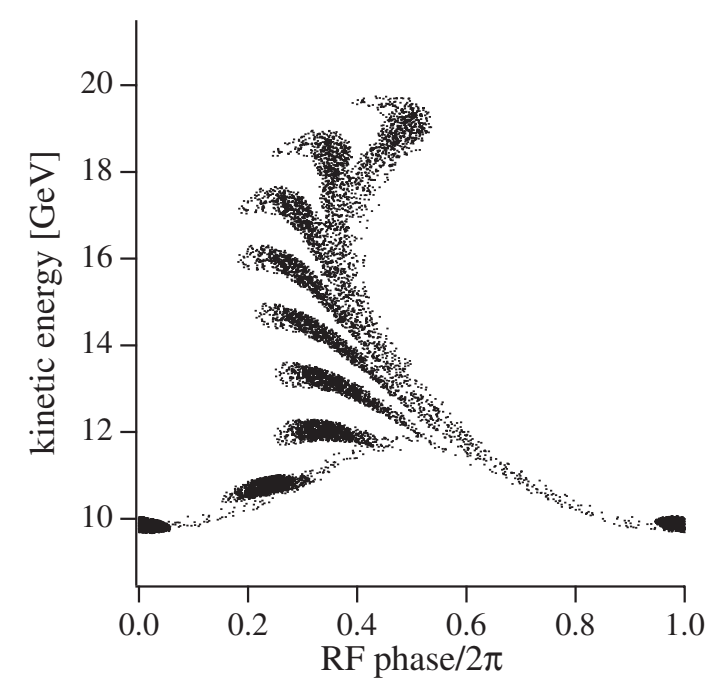

(b)

FIG. 4. Longitudinal emittance evolution with different initial distribution. (a) starts from 6D ellipsoidal distribution and (b) starts from uncorrelated distribution in horizontal, vertical, and longitudinal planes. 
solenoid and other vacuum components, one may assume that a correlation between horizontal and vertical space is created on the way. On the other hand, there seems to be nothing upstream which generates any noticeable coupling between longitudinal and transverse space. In the following studies, we assume the distribution in Fig. 3(b).

\section{POSSIBLE CURES}

The blowup occurs because energy gain is a function of rf phase whereas time of flight depends on transverse amplitude. Resulting phase slip introduces the energy spread at the end. To cure the problem, the following methods are proposed and examined by simulations. The most naive method is to increase rf voltage so that one can finish acceleration before phase slip and therefore energy spread are accumulated. The second method is to use a higher harmonic rf. The higher harmonic rf voltage added to the fundamental one makes the rf crest flatter so that energy gain becomes less sensitive to rf phase.

One-thousand macroparticles are prepared with longitudinal emittance of $0.5 \mathrm{eV}$ s and shaped according to Berg's criterion [13]. Transversely, they are located in a 4D ellipsoidal phase space and the distribution is Waterbag. There is no correlation between longitudinal and transverse distribution. For the lattice, a muon acceleration ring from 10 to $20 \mathrm{GeV}$ is modeled. A $100 \%$ nominal normalized emittance is $30 \pi \mathrm{mm}$ rad.

Figure 5 shows the final momentum spread as a function of initial transverse emittance taking rf voltage as a parameter. The case with the nominal rf voltage shows that there is a quite large increase of $d p / p$ as a function of transverse emittance which was seen in Fig. 3(b) previously. A $50 \%$ increase of $\mathrm{rf}$ voltage improves transverse emittance dependence. Little further improvement is achieved even if the voltage is increased to twice as

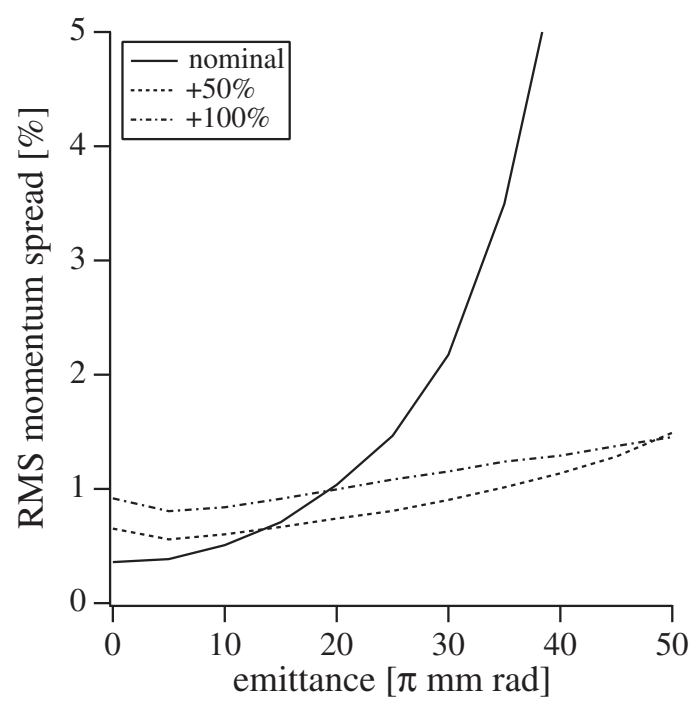

FIG. 5. Final momentum spread as a function of initial transverse emittance with different accelerating voltages.

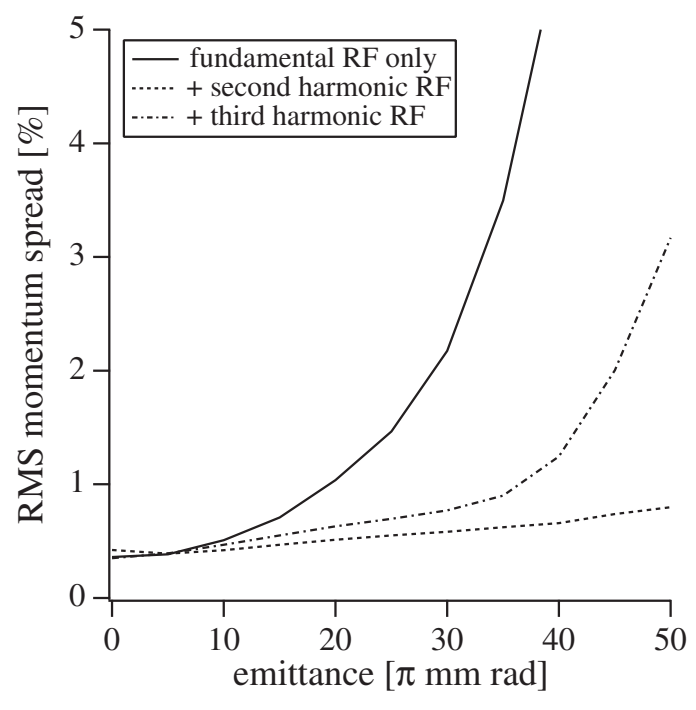

FIG. 6. Final momentum spread as a function of initial transverse emittance with and without higher harmonic rf.

much. Figure 6 is a similar figure but the parameter is now higher harmonic rf. Adding a second harmonic rf makes the voltage flat around the crest. A third harmonic rf also flattens the voltage but with a smaller phase region. The figure shows that the second harmonic rf almost eliminates the increase of momentum spread and the third harmonic rf does up to $40 \pi \mathrm{mm}$ rad. Obviously, the wider range of acceptable emittance achieved with second harmonic rf compared with the third harmonic can be attributed to the greater flatness in rf phase. Incidentally, the voltage required to make the crest flat with second and third harmonic rf is $1 / 4$ and $1 / 9$ of the fundamental $\mathrm{rf}$ voltage, respectively.

\section{FFAG CASCADE}

A scheme to use FFAG as a muon accelerator assumes a FFAG cascade will be employed because the ratio of injection to extraction momenta in nonscaling FFAG is limited to about two. For example, the first FFAG accelerates muons from 5 to $10 \mathrm{GeV}$ and the second from 10 to $20 \mathrm{GeV}$. Detailed design parameters can be obtained in [12]. Matching between two FFAGs can be, in principle, done in longitudinal and transverse space provided the transverse emittance is small. Figure 7 shows the longitudinal emittance evolution and the momentum spread of both FFAGs together when the transverse emittance is zero. There should be an appropriate rf phase shift between the first and second FFAG.

The longitudinal emittance blowup again becomes serious and not many particles are accelerated to the final energy when one takes $100 \%$ transverse emittance of $30 \pi \mathrm{mm}$ rad as shown in Fig. 8(a). The same cures can be applied and Fig. 8(b) is the best optimized scenario so far for the FFAG cascade. Second harmonic rf voltage is incorporated and both fundamental and second harmonic 


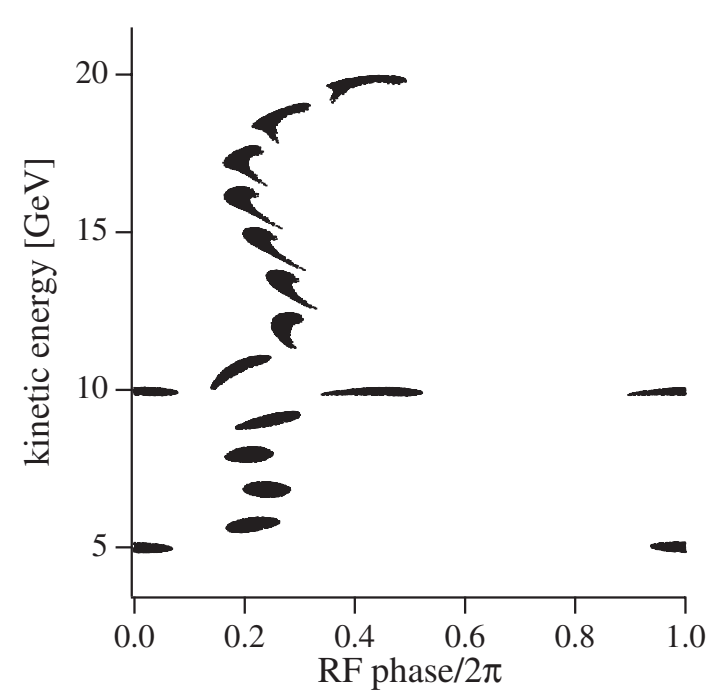

(a)

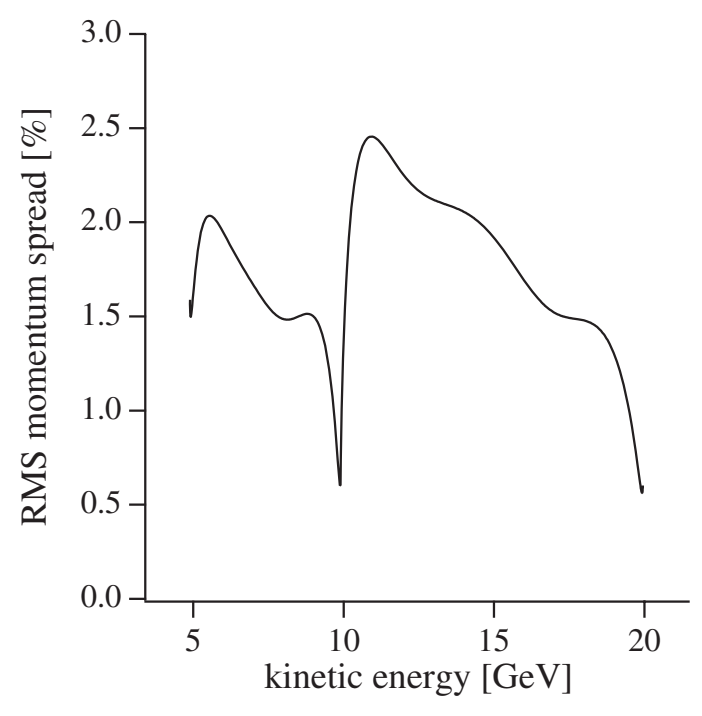

(b)

FIG. 7. (a) Longitudinal emittance evolution and (b) momentum evolution of a two FFAG cascade. Transverse emittance is zero.

voltages are increased by $10 \%$. Phase space looks much better than before. Since beam loss is inevitable in this case, we introduced allowed momentum spread as another measure instead of rms momentum spread and see how the beam loss relates to it at the final energy in Fig. 9.

\section{DISCUSSIONS AND SUMMARY}

Potentially, in any accelerator, there is a path length difference due to transverse amplitude. Although it is not noticeable in an ordinary accelerator, it appears a limiting factor of transverse acceptance in muon FFAG for the following reasons. First, the beam emittance is huge compared with an ordinary accelerator. It is obvious to say that the difference in path length is exacerbated in the case of a large emittance beam. Second, it is also due to the method of acceleration in nonscaling FFAG. A particle is accelerated out of the rf bucket and no synchrotron oscillation takes place. If there are synchrotron oscillations, a rigid $\mathrm{rf}$ phase relation can be avoided and the final momentum spread can be reduced.

Two methods of curing the problem seem to work for a single FFAG system. It can be almost eliminated if one increases rf voltage. However, a FFAG with very high

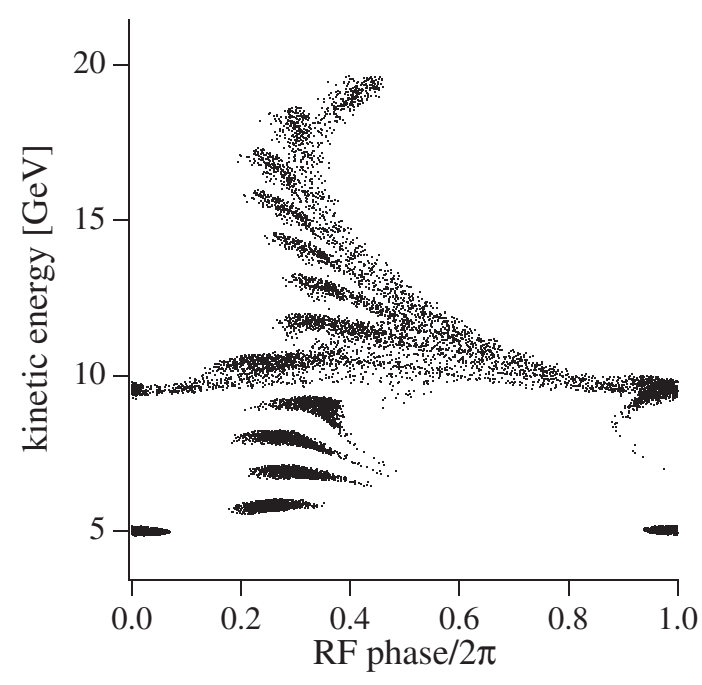

(a)

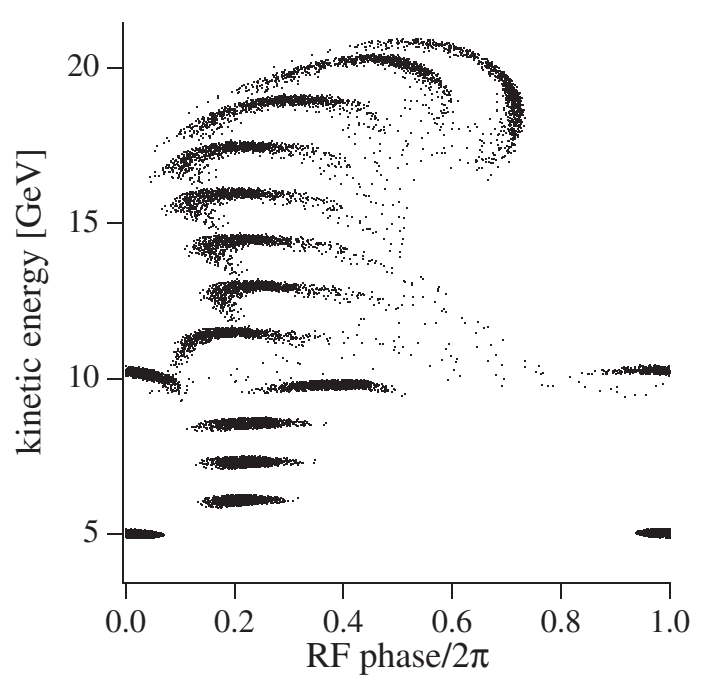

(b)

FIG. 8. Longitudinal emittance evolution of a two FFAG cascade with $100 \%$ transverse emittance of $30 \pi \mathrm{mm}$ rad. (a) Nominal rf voltage and no higher harmonic rf. (b) Second harmonic rf added and 10\% increase of rf voltage. 


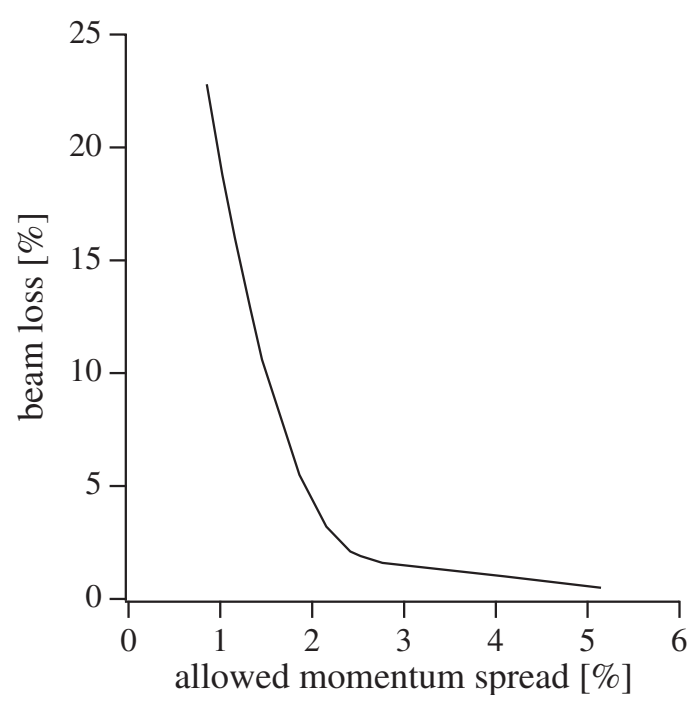

FIG. 9. Beam loss as a function of allowed momentum spread. If a muon storage ring downstream has enough momentum acceptance, beam loss becomes smaller.

voltage would be counterproductive because one of main advantages of a FFAG is that in using the rf cavity several times the voltage can be moderate and the cost lower than in any other type of accelerator. The voltage should be as low as possible to optimize the total cost.

Use of higher harmonic rf reduces the final momentum spread because the energy gain becomes less sensitive to rf phase. However, the spread in phase direction cannot be eliminated completely.

The problem is more pronounced in a FFAG cascade. With higher voltage or higher harmonic rf, the longitudinal emittance blowup in the first FFAG can be partially cured. However, a spread in phase as a result of difference of time of flight depending on transverse emittance remains. More phase spread at the entrance of the second FFAG introduces further spread during the acceleration. Again the same solutions, namely of higher voltage and of higher harmonic rf, help, but now partial particle loss is inevitable.

\section{ACKNOWLEDGMENTS}

The work is supported by the U.K. Neutrino Factory/ Particle Physics and Astronomy Research Council (PPARC) under Contract No. 2054. I would like to thank Dr. C. Prior and Dr. D. Kelliher for helpful comments on the manuscript.

[1] S. Machida, Nucl. Instrum. Methods Phys. Res., Sect. A 503, 41 (2003).

[2] M. Aiba, K. Koba, S. Machida, Y. Mori, R. Muramatsu, C. Ohmori, I. Sakai, Y. Sato, A. Takagi, R. Ueno, T. Yokoi, M. Yoshimoto, and Y. Yuasa, Proceedings of EPAC 2000, Vienna, p. 581.

[3] C. Johnstone, W. Wan, and A. Garren, Proceedings of PAC 1999, New York, p. 3068.

[4] A. A. Kolomensky and A. N. Lebedev, Theory of Cyclic Accelerators (North-Holland, Amsterdam, 1966).

[5] "The Neutrino Factory and Muon Collider Collaboration Study 2-A," http://www.cap.bnl.gov/mumu/study2a/.

[6] For example, E. Keil and A.M. Sessler, Nucl. Instrum. Methods Phys. Res., Sect. A 538, 159 (2005); D. Trbojevic, E. D. Courant, and M. Blaskiewicz, Phys. Rev. ST Accel. Beams 8, 050101 (2005).

[7] C. Johnstone and S. Koscielniak, Nucl. Instrum. Methods Phys. Res., Sect. A 503, 445 (2003).

[8] S. Koscielniak and C. Johnstone, Nucl. Instrum. Methods Phys. Res., Sect. A 523, 25 (2004).

[9] E. D. Courant, J.S. Berg, D. Trbojevic, R. Talman, and A. A. Garren, Proceedings of PAC 2003, Portland, p. 3485.

[10] E. Forest, F. Schmidt, and E. McIntosh, KEK Report 2002-3 and also CERN-SL-2002-044 AP.

[11] J.S. Berg, Proceedings of the International Workshop on FFAG Accelerators, Osaka, 2005, p. 73.

[12] "International Scoping Study Machine Working Group U.S. FFAG Specifications," http://www.cap.bnl.gov/ mumu/projects/ISS/accel/us-ffag.html.

[13] J. S. Berg, Phys. Rev. ST Accel. Beams 9, 034001 (2006). 